\title{
Nutrição e neurocirurgia: uma revisão
}

\author{
Bárbara Pelicioli Riboldi', Betânia Contini' ${ }^{1}$, Fernanda Trespach dos Santos ${ }^{1}$, \\ Laís Souza da Silva1, Vanessa Rossoni de Oliveira1', Fernanda Monte da \\ Cunha ${ }^{1}$, Paola Maria Brolin Santis-Isolan², Gustavo Rassier Isolan ${ }^{3}$ \\ Universidade Federal do Rio Grande do Sul (UFRS)
}

\section{RESUMO}

As alterações metabólicas e da composição corporal em decorrência da injúria cirúrgica resultam em necessidades nutricionais especiais para pacientes neurocirúrgicos. Essas alterações têm como característica a instalação de um estado hipermetabólico e hipercatabólico, observando-se uma redução nas reservas corporais de lipídeos e proteínas, resultando na dificuldade de cicatrização e risco aumentado de infecção. Além disso, com um aporte nutricional inadequado, os pacientes podem evoluir para um estado de desnutrição. Na ausência de diretrizes específicas de como o suporte nutricional deve suceder em traumas neurológicos, verificou-se a importância da realização de um artigo de revisão a respeito do suporte nutricional utilizado em pacientes neurocirúrgicos. A partir da pesquisa realizada no banco de dados Medline (1976-2010), observou-se que a estimativa energética é um fator decisivo no prognóstico do paciente, a suplementação de aminoácidos não se mostra necessária e que, além dos exames de rotina, devem-se realizar exames de eletrólitos e osmolalidade urinários e plasmáticos. O suporte nutricional ideal parece ser uma administração conjunta de nutrição enteral e parenteral e, quanto mais precocemente essa alimentação é iniciada, melhores são os desfechos clínicos. Considerando que é de competência do nutricionista a prescrição dietética da nutrição enteral, sua presença é essencial na equipe multiprofissional de suporte nutricional.

\section{PALAVRAS-CHAVE}

Nutrição, neurocirurgia, nutrição de grupos de risco, nutrição enteral, nutrição parenteral.

\begin{abstract}
Nutrition and neurosurgery: a review

The metabolic and body composition alterations as a result of neurosurgical injury results in special dietary needs for neurosurgical patients. These changes are characterized by the installation of a hypermetabolic and hypercatabolic states, observing a reduction in body reserves of lipids and proteins. The scenario results in poor wound healing and an increased risk of infection. Moreover, with an inadequate nutritional support, patients can develop malnutrition. In the absence of specific guidelines of nutritional support in neurosurgical patients the authors verified the importance in make a review article about nutrition in neurosurgical patients. From the survey conducted in Medline (1976-2010), we observed that the estimated energy is a decisive factor in the patient prognosis, on the other hand, the amino acid supplementation does not seem necessary. It is necessary to conduct examinations of urinary and plasma electrolytes and osmolality. The ideal nutritional support appears to be a combination of precocious enteral and parenteral nutrition in severe neurological status patients to improve clinical outcomes. Whereas it is the responsibility of the nutritionist's dietary prescription, their presence is essential in the multidisciplinary team of nutritional support.
\end{abstract}

\section{KEYWORDS}

Nutrition, neurosurgery, nutrition risk groups, enteral nutrition, parenteral nutrition.

\section{Introdução}

Desde 1930, têm-se discutido as alterações metabólicas e de composição corporal em decorrência de injúria cirúrgica. Sabe-se que o metabolismo energético bem como o metabolismo dos fluidos, eletrólitos e a função endócrina são afetados. Essas alterações são percebidas pela perda de peso, diminuição da força e atividade muscular e declínio temporário da capacidade intelectual desses pacientes. ${ }^{5}$

Pacientes neurocirúrgicos apresentam necessidades especiais, uma vez que, logo após o insulto

1. Acadêmicas do curso de Nutrição da Universidade Federal do Rio Grande do Sul (UFRGS).

2. Professora adjunta do Departamento de Cirurgia (Cirurgia Pediátrica) do Hospital de Clínicas de Porto Alegre da UFRGS, regente da disciplina de nutrição da UFRGS.

3. Professor permanente do curso de pós-graduação em Medicina: Ciências Cirúrgicas (Hospital de Clínicas de Porto Alegre) da UFRGS. 
neurológico crítico, o estado hipermetabólico e hipercatabólico está evidente. Esse estado hipermetabólico leva a uma redução nas reservas corporais de lipídeos e proteínas, resultando em dificuldade de cicatrização e risco aumentado de infecção. Além disso, com aporte nutricional inadequado, os pacientes podem evoluir para um estado de desnutrição que pode ser resultante da alimentação insuficiente, de necessidade energética maior ou de alterações da utilização dos nutrientes. ${ }^{21}$

Por causa do trauma desses pacientes neurocirúrgicos, a avaliação nutricional torna-se um grande desafio para a equipe de saúde, uma vez que, previamente à cirurgia, os pacientes encontram-se eutróficos, mudando drasticamente para o estado de desnutrição proteico-calórica pós-cirurgia. ${ }^{10}$

Não existem diretrizes específicas de como o suporte nutricional deve suceder em traumas neurológicos, porém alguns estudos têm mostrado que uma combinação da nutrição enteral e da parenteral, quando administradas em até 48 horas após o procedimento, tem sido associada com efeitos benéficos como a atenuação da resposta hipercatabólica, da atrofia intestinal, da perda muscular e da infecção. Entretanto, a existência de desafios como a alteração da motilidade gastrointestinal, o aumento da pressão intracraniana e o comprometimento do estado de consciência e da função neurológica geral pode resultar no atraso desses procedimentos e, assim, na piora do prognóstico. ${ }^{30}$

Com o objetivo de apresentar um artigo de revisão a respeito do suporte nutricional utilizado em pacientes neurocirúrgicos, foi realizada uma pesquisa no banco de dados Medline incluindo estudos de 1976 a 2010.

\section{Estimativas energéticas}

Estudos têm sido realizados na tentativa de definir uma fórmula que estime de forma mais precisa as necessidades energéticas dos pacientes neurocirúrgicos, a fim de obter adequado suporte nutricional e melhor prognóstico. Não existe consenso a respeito da maneira mais apropriada de administração da dieta e da necessidade de restrição hídrica nesse quadro. ${ }^{5}$

Em virtude do estado catabólico em que os pacientes neurocirúrgicos se encontram, suas necessidades calóricas apresentam-se aumentadas, podendo atingir de $120 \%$ a $250 \%$ da taxa metabólica basal (TMB), enquanto nos pacientes imobilizados o gasto varia entre $100 \% \mathrm{e}$ $120 \% .{ }^{5}$ Clifton et al. (1984) desenvolveram duas fórmulas para estimativa do aumento em percentual da TMB desses indivíduos, tendo em vista a frequência cardíaca, a pressão arterial (PA), a temperatura corporal, dias pós-injúria (DPI) e escala de coma de Glasgow (ECG). Até duas semanas após o dano, em pacientes com ECG $\leq 7$, considera-se $\mathrm{TMB}=152-14(\mathrm{ECG})+0,4(\mathrm{PA})+$ 7 (DPI), já para os indivíduos com ECG $\geq 8, \mathrm{TMB}=$ $90-3$ (EGC) + 0,9 (PA). ${ }^{6}$

Sunderland e Heibrun (1992) compararam três diferentes fórmulas para cálculo da TBM - Harris \& Benedict, Clifton e uma outra fórmula baseada no estresse metabólico - com o método da calorimetria indireta. Todas as fórmulas obtiveram diferenças significativas e discrepantes em relação à calorimetria, atentando para os cuidados que devem ser tomados na avaliação nutricional. Tendo em vista que muitos hospitais não dispõem de equipamento para calorimetria na assistência nutricional, as equações são formas rotineiras de estimativa energética, que, se calculadas erroneamente, podem levar a super ou subalimentação. ${ }^{26}$

Algumas diretrizes atuais definem que a principal recomendação é atingir as necessidades energéticas até sete dias pós-injúria para redução da mortalidade. São estimados $140 \%$ da TMB para pacientes não imobilizados e $100 \%$ da TMB para pacientes imobilizados. ${ }^{27}$ Para que esse objetivo seja atingido, recomenda-se iniciar o suporte nutricional dentro de no máximo $72 \mathrm{~h}$ pós-injúria. ${ }^{28} \mathrm{Se}$ o consumo calórico total não for atingido por um período de duas semanas após a lesão, o risco de mortalidade estará aumentado drasticamente. ${ }^{12,17,23-25}$

\section{Ingestão precoce}

Harlt et al. (2008) mostraram que pacientes que não receberam alimentação durante sete dias após a injúria apresentaram risco quatro vezes maior de mortalidade nas primeiras duas semanas, ${ }^{15}$ verificando-se, assim, a grande importância da ingestão precoce. Entretanto, Young et al. (1987) observaram que a substituição da alimentação via parenteral precoce por enteral tardia não mostrou variações na morbidade. ${ }^{29}$

\section{Ingestão proteica}

Após uma lesão cerebral grave, há aumento da requisição de energia e da excreção de nitrogênio. ${ }^{14}$ A perda média de nitrogênio desses pacientes é o dobro ou o triplo de um paciente normal e poderá ocasionar uma redução de $10 \%$ na massa magra em sete dias, podendo acarretar desnutrição, que, se mantida por duas a três semanas, resultará em uma perda de peso de até $30 \% .{ }^{11}$ Em virtude do percentual de proteína das 
formulações enteral e parenteral - máximo $20 \%$-, o equilíbrio de nitrogênio raramente é alcançado. No entanto, quanto maior o teor de nitrogênio dos alimentos consumidos, maior sua retenção. ${ }^{7}$

Oertel et al. (2009) não encontraram resultados significantes na administração do dobro de aminoácidos recomendado em diretrizes (15\% da ingestão energética total) em uma combinação de nutrição enteral e parenteral, porém o grupo tratado apresentou maior valor sérico de albumina. Eles relatam que esse excesso de aminoácidos estaria sendo desaminado e/ou desviado para as rotas de oxidação, uma vez que os pacientes tratados apresentaram valores maiores de transaminase glutâmica oxalacética (TGO) e transaminase glutâmica pirúvica (TGP). ${ }^{21}$

\section{Desequilíbrio hidreletrolítico}

A hiponatremia é a alteração eletrolítica mais relatada em pacientes neurocirúrgicos. Pode aparecer por meio da SIADH (síndrome da secreção inapropriada do hormônio antidiurético), que leva ao aumento do volume intravascular, visto que o $\mathrm{ADH}$ causa retenção hídrica; ou também pela depleção de sódio cerebral, mediante a perda renal, com diminuição do volume plasmático. $^{9}$

Os sintomas da forma leve de hiponatremia incluem anorexia, dores de cabeça, irritabilidade e fraqueza muscular. Já quando severa, a hiponatremia pode levar a edema cerebral, excitabilidade neuromuscular, espasmos e câimbras musculares, náuseas e vômitos e confusão, e ainda é possível o desenvolvimento de dano neurológico permanente, coma ou morte.

A hiponatremia causada pela SIADH é tratada com restrição de fluidos. Se crônica e minimamente sintomática, pode ser revertida lentamente por restrição hídrica leve, de 1.200 a $1.800 \mathrm{ml} / \mathrm{d}$. Já a forma aguda deve ser prontamente tratada, visto que tem sido associada com edema cerebral; esse tratamento se dá por uma restrição hídrica mais significativa, de $<1$ 1/d; caso se manifeste de forma severa ou sintomática, recomenda-se a utilização de solução salina hipertônica. Já a depleção de sódio cerebral causada por perda renal de sódio é revertida com reposição de sódio e fluidos, podendo ser exacerbada por restrição hídrica. ${ }^{27}$

Ainda, o adiamento da reinserção da alimentação (NPO por diversas horas, que era frequentemente utilizado na prática clínica) pode estimular a secreção de $\mathrm{ADH}$, que pode persistir após o estresse cirúrgico. Clark (1971) propõe a necessidade da monitoração de rotina dos eletrólitos e osmolalidade urinários e plasmáticos em pacientes neurocirúrgicos. ${ }^{5}$

\section{Nutrição enteral}

Segundo a Sociedade Europeia de Nutrição Clínica e Metabolismo, a nutrição enteral é recomendada preferencialmente à nutrição parenteral para pacientes em estado crítico, uma vez que a alimentação por sonda nasogástrica é associada com melhores resultados, pois mantém a permeabilidade intestinal, e a translocação bacteriana é menor. ${ }^{17}$ Porém, a dificuldade do uso da nutrição enteral em pacientes neurocirúrgicos é exacerbada, uma vez que muitos se encontram inconscientes e necessitam manter-se deitados no período pré e pós-cirúrgico, correndo o risco de haver aspiração e consequentemente pneumonia aspirativa, que possui alta taxa de mortalidade. ${ }^{10}$

Norton et al. (1988) encontraram uma relação significativa do tempo que os indivíduos levaram para tolerar totalmente a dieta enteral (em concentração e volume estipulados) com os níveis elevados de pressão intracraniana e escores diminuídos na escala de coma de Glasgow, ${ }^{20}$ mostrando, assim, que a tolerância à terapia nutricional enteral está associada à melhora do quadro clínico desses pacientes.

Kao et al. (1998) mostraram que, após o traumatismo cranioencefálico (TCE), mais de $50 \%$ dos pacientes não toleram a nutrição enteral. Também foi relatada a ocorrência de vômitos, dor abdominal, distensão e aumento do volume gástrico residual. ${ }^{16}$ Têm-se observado, ainda, redução das células epiteliais, atrofia e edema no tecido intersticial das vilosidades. ${ }^{3}$ Uma das únicas relações estabelecidas até o momento entre o cérebro e o sistema gastrointestinal envolve a pressão intracraniana e a contratilidade gástrica, e com o aumento da pressão há redução, em até $80 \%$, da contratilidade gástrica. ${ }^{22,19}$

Zarbock et al. (2008) buscaram uma relação da gravidade do estado neurológico com o aporte nutricional e concluíram que a severidade do estado não afeta o aporte energético nem o dia de início da nutrição enteral. Além disso, constataram que o atraso do início da terapia nutricional se dá por razões como a colocação do tubo de alimentação, o requerimento de radiografia abdominal para verificar se o tubo está no local correto e a aquisição da nutrição enteral. ${ }^{30}$

Diretrizes indicam administração de soluções isotônicas inicialmente a $30 \mathrm{ml} / \mathrm{h}$, aumentando em $15-25 \mathrm{ml}$ a cada $12 \mathrm{~h}-24 \mathrm{~h}$, de acordo com a tolerância do paciente, verificando-se a presença de resíduos gástricos. ${ }^{27}$

\section{Nutrição parenteral}

Quando a terapia nutricional ocorre apenas pela nutrição parenteral, consequências como hipertrofia 
das vilosidades intestinais, superalimentação e hiperglicemia têm sido relatadas, assim como redução da secreção das enzimas dissacaridases no trato gastrointestinal, que pode conduzir a aumento do risco de sepse. Sendo assim, a nutrição parenteral, como única terapia nutricional, parece ser menos benéfica para pacientes neurocirúrgicos.

Pacientes com injúria cerebral sofrem de estresse catabólico intenso após o trauma. Rapp et al. (1983) encontraram maior sobrevida nos pacientes que receberam nutrição parenteral logo após a injúria (48h) quando comparada à nutrição enteral. Esse fato deve-se principalmente à ineficiência do trato gastrointestinal logo após o dano cerebral. Ainda, os pacientes que receberam nutrição parenteral tiveram elevação do escore de coma de Glasgow ( $p<0,001)$, balanço nitrogenado maior $(\mathrm{p}<0,002)$ e não taxa de mortalidade nula ao longo dos dias de tratamento (18 dias). ${ }^{24}$

\section{Nutrição enteral e parenteral}

Nenhuma diferença significativa nos níveis séricos de albumina, perda de peso, balanço nitrogenado, taxa de infecção ou desfecho final foi encontrada entre as formas de administração enteral e parenteral. ${ }^{14}$

Entre as vantagens da nutrição enteral, observam-se menor risco de hiperglicemia e de infecção e custo reduzido. ${ }^{13}$ No entanto, pressão intracraniana aumentada e gravidade da lesão cerebral podem adiar o início da nutrição enteral. ${ }^{20}$ Esse atraso na alimentação foi atribuído a aumento residual gástrico, atraso no esvaziamento gástrico, pneumonia aspirativa e diarreia. ${ }^{3,24} \mathrm{Em}$ tais circunstâncias, a nutrição parenteral total é muitas vezes recomendada.

Ott et al. (1991) e Rapp et al. (1983) acreditam que o suporte nutricional parenteral precoce melhora o resultado após a injúria. ${ }^{22,24}$ Young et al. (1987), Hadley et al. (1986) e Borzotta et al. (1994) comprovam que, com quantidades equivalentes de alimentação, o modo de administração não tem efeito sobre resultado neurológico, ou seja, tanto a nutrição parenteral como a enteral são igualmente eficazes quando prescritas individualmente de acordo com o gasto de energia e nitrogênio excretado. ${ }^{4,14,29}$

\section{Equipe multiprofissional}

A importância da atuação de uma equipe hospitalar multiprofissional, principalmente no que se refere à recuperação nutricional dos pacientes, já vem sendo bem valorizada, visto que a desnutrição é frequente em hospitalizados. Martens et al. (1990) relataram o impac- to de um programa de tratamento multidisciplinar em pacientes neurológicos com disfagia, uma consequência comum neles. Com a atuação de fisioterapeutas, nutricionistas, enfermeiros e médicos, o grupo tratado obteve maior ingestão calórica, bem como ganho de peso e melhora do estado nutricional. ${ }^{18}$

Anette e Wenströn (2005) realizaram questionário com profissionais da área da saúde (auxiliares de enfermagem, enfermeiros e médicos, não considerando a presença do nutricionista na equipe) após a implementação de diretrizes acerca dos cuidados nutricionais em uma unidade de terapia intensiva. Os autores encontraram divergências de opiniões entre os profissionais, além da falta de conhecimento sobre cuidados nutricionais relativos às recomendações estabelecidas. As diretrizes continham áreas que deveriam ser aperfeiçoadas, a fim de melhorar o atendimento nutricional ao paciente $\mathrm{e}$ também a integração da equipe para que haja melhor diálogo, tendo em vista a importância de uma equipe multiprofissional na tomada de decisões conjuntas a respeito de intervenções adequadas de acordo com a situação do paciente. ${ }^{2}$

\section{Comentários finais}

A estimativa energética é um fator decisivo no prognóstico do paciente. Tendo em vista a falta de equipamentos de calorimetria, é necessário avaliar as diferentes possibilidades de estimativas e, a partir de uma avaliação nutricional detalhada, escolher a que apresenta ser mais adequada.

Não é necessária a suplementação de aminoácidos na terapia nutricional que combine nutrição enteral e parenteral, quando administrada em até 48 horas após o insulto neurológico.

Além da monitoração de exames de rotina, é necessária também a realização de exames de eletrólitos e osmolalidade urinários e plasmáticos para diagnosticar uma possível hiponatremia.

O suporte nutricional dependerá exclusivamente do estado de consciência e tolerabilidade do paciente. O ideal parece ser a administração conjunta de nutrição enteral e parenteral, e quanto mais precocemente esse suporte nutricional for iniciado, melhores serão os desfechos clínicos. Se somente um suporte parenteral total for possível, a equipe deve ter atenção para avaliar um risco maior de infecção, translocação bacteriana e sub ou superalimentação.

Segundo a legislação brasileira, compete ao nutricionista a prescrição dietética da nutrição enteral, logo é essencial sua presença na equipe multiprofissional de suporte nutricional. ${ }^{1}$ Para que a terapia nutricional 
ocorra de forma adequada e no tempo mínimo de início, os cuidados devem ser tomados pela equipe que providenciará a terapia, sendo de suma importância a integralização dos profissionais envolvidos nos cuidados desses pacientes.

\section{Referências}

1. Agência Nacional de Vigilância Sanitária. Resolução RDC $\mathrm{n}^{\circ} 63$, de 6 de julho de 2000.

2. Anette $\mathrm{H}$, Wenströn $\mathrm{Y}$. Implementing clinical guidelines for nutrition in a neurosurgical intensive care unit. Nurs Health Sci. 2005;7:266-72

3. Aydin S, Ulusoy H, Usul H, Yulug E, Cobanoglu U, Aydin K, et al. Effects of early versus delayed nutrition on intestinal mucosal apoptosis and atrophy after traumatic brain injury. Surg Today. 2005;35(9):751-9.

4. Borzotta AP, Pennings J, Papasadero B, Paxton J, Mardesic S, Borzotta R, et al. Enteral versus parenteral nutrition after severe closed head-injury. J Trauma. 1994;37(3):459-68.

5. Clark RG. Caloric requirements after operation. Proc Nutr Soc. 1971;30:158-65.

6. Clifton GL, Robertson CS, Grossman RG, Hodge S, Foltz R, Garza C. Metabolic response to severe head injury. J Neurosurg. 1984;60:687-96.

7. Clifton GL, Robertson CS, Constant DF. Enteral hyperalimentation in head injury. J Neurosurg. 1985;62:186-93.

8. Clifton GL, Robertson CS, Choi SC. Assessment of nutritional requirements of head-injured patients. J Neurosurg. 1986;64:895-901.

9. Diringer M, Ladenson PW, Borel C, Hart GK, Kirsch JR, Handley DF. Sodium and water regulation in a patient with cerebral salt wasting. Arch Neurol. 1989;46:928-30.

10. Duarte ACG. Avaliação nutricional: aspectos clínicos e laboratoriais. $1^{\text {a }}$ ed. São Paulo: Atheneu; 2007. p (il).

11. Duke JH, Jorgensen SD, Broell JR, Long CL, Kinney JM. Contribution of protein to caloric expenditure following injury. Surgery. 1970;68:168-74.

12. Ghanbari CRD. Protocols for nutrition support of neuro intensive care unit patients: a guide for residents. Internet J Emerg Intensive Care Med. 1999;3(1):1-13.

13. Glaesener JJ, Fredebohm M. Percutaneous endoscopic gastrostomy in the rehabilitation of neurological disorders. J Suisse Med. 1992;122(43):1600-5.

14. Hadley MN, Grahm TW, Harrington T, Schiller WR, McDermott MK, Posillico DB. Nutritional support and neurotrauma: a critical review of early nutrition in forty-five acute head injury patients. Neurosurgery. 1986;19:367-73.

15. Harlt R, Gerber L, Ni Q, Ghajar J. Effect of early nutrition on deaths due to severe traumatic brain injury. J Neurosurg. 2008;109:50-6.
16. Kao $\mathrm{CH}$, Changlai SP, Chieng PU, Yen TC. Gastric emptying in head-injured patients. Am J Gastroenterol. 1998;93(7):1108-12.

17. Kreymann KG, Berger MM, Deutz NE, Hiesmayr M, Jolliet $P$, Kazandjiev $\mathrm{G}$, et al. ESPEN guidelines on enteral nutrition: intensive care. Clin Nutr. 2006;25:210-23.

18. Martens L, Cameron T, Smonsen M. Effects of a multidisciplinary management program on neurologically impaired patients with dysphagia. Dysphagia. 1990;5(3):147-51.

19. Minami $\mathrm{H}$, McCallum RW. The physiology and pathophysiology of gastric emptying in humans. Gastroenterology. 1984;86:1592-610.

20. Norton JA, Ott LG, McClain C, Adams L, Dempesey RJ, Haack $D$, et al. Intolerance to enteral feeding in the braininjured patient. J Neurosurg. 1988;68(1):62-6.

21. Oertel MF, Hauenschild A, Gruenschilaeger J, Muller B, Scharbrodt W, Boeker DK. Parenteral and enteral nutrition in the management of neurosurgical patients in the intensive care unit. J Clin Neurosci. 2009;16:1161-7.

22. Ott L, Young B, Phillips R, McClain C, Adams L, Dempesey $R$, et al. Altered gastric emptying in the head-injured patient: relationship to feeding intolerance. J Neurosurg. 1991;74(5):738-42.

23. Qiu WS, Liu WG, Shen H, Wang WM, Hang ZL, Zhang Y, et al. Therapeutic effect of mild hypothermia on severe traumatic head injury. Chin J Traumatol. 2005;8:27-32.

24. Rapp RP, Young B, Twyman D, Bivins BA, Haack D, Tibbs PA. The favorable effect of early parenteral feeding on survival in head injured patients. J Neurosurg. 1983;58(6):906-12.

25. Singer P, Berger MM, Van den Berghe G, Biolo G, Calder P, Forbes $A$, et al. ESPEN guidelines on parenteral nutrition: intensive care. Clin Nutr. 2009;28:387-400.

26. Sunderland PM, Heibrun M. Estimating energy expenditure in traumatic brain injury: comparison of indirect calorimetry with predictive formulas. Neurosurgery. 1992;31(2):246-53.

27. Sunderland PM, Heibrun M. Handbook of neurosurgery. $5^{\mathrm{a}}$ ed. Lakeland, Fla: Thieme; 2001. p (il).

28. Twyman D. Nutritional management of the critically ill neurologic patient. Crit Care Clin. 1997;13:39-49.

29. Young B, Ott L, Twyman D, Norton J, Rapp R, Tibbs P, et al. The effect of nutritional support on outcome from severe head injury. J Neurosurg. 1987;67:668-76.

30. Zarbock SD, Steinke D, Hatton J, Magnuson B, Smith KM, Cook AM. Successful enteral nutritional support in the neurocritical care unit. Neurocritic Care. 2008;9:210-6.

\section{Endereço para correspondência}

Gustavo Rassier Isolan

Rua Ramiro Barcelos, 2400, $2^{\circ}$ andar, Bairro Bom Fim

90035-003 - Porto Alegre, RS

Telefone: (51) 3308-5607/Telefax: (51) 3308-5617

E-mail: ppgcirur@ufrgs.br 